Heredity (1978), 41 (3) 407

\title{
GENETIC PARAMETER ESTIMATION: A GENERAL-PURPOSE MAXIMUM LIKELIHOOD PROGRAM
}

Received 25.vii.78

I have available copies of a document with the above title, describing a program, written in SNOBOL4 for estimating such parameters as genefrequencies, average inbreeding and recombination values. Copies of this, about 18 pages of $\mathrm{A} 4$, are available from me at the address below, as are copies of the program.

JOHN ROSTRON

Department of Biology,

North East London Polytechnic,

Romford Road,

London E15 $4 \mathrm{LZ}$ 